\title{
Odysseia: Genetic Regulatory Feature Analysis with Interpretable Classification Machine Learning Models
}

\author{
Gang $\mathrm{Yu}^{1, * 1}$, Jiawang Tao ${ }^{1,}$, and Jie Wang ${ }^{1, * 2}$ \\ ${ }^{1}$ Center for Health Research, Guangzhou Institutes of Biomedicine and Health, Chinese Academy of Sciences, \\ Guangzhou 510530, China \\ ${ }^{* 1}$ Correspondence: gyu17@alumni.jh.edu \\ ${ }^{* 2}$ Correspondence: wang_jie01@gibh.ac.cn
}

\begin{abstract}
With rapid progress of robust single-cell transcriptome sequencing since last decade, numerous complex mechanisms underlying cell development has been revealed. Single-cell RNA sequencing (scRNA-seq) analysis is widely accepted as the main approach to define cell stages and phenotypes. As conversion of somatic cells into induced pluripotency cells succeeded, identification key genetic factors(GFs) with scRNA-seq for cell reprogramming in biological research and regenerative medicine fields gained increasing attention. Herein, we describe Odysseia, an interpretable machine learning classifier based single-cell gene expression profile(scGEP) analysis system, that assesses importances of genetic regulatory features in differentiating cell states(CSs). Furthermore, extracted factors, when combining with regulatory network analysis, can help to find key GFs in classifying CSs and possibly inducing CS conversions. Analyzed three published scRNA-seq datasets used to study divergent cell types, Odysseia correctly extracted GFs acclaimed to be capable of inducing CS conversions. Overall, Odysseia provides an automated alternative to obtain guidance information while explicating mechanism to engineer cellular phenotypes.
\end{abstract}

\section{Introduction}

Since cell reprogramming of mouse embryonic fibroblasts(MEF) to induced pluripotent stem cell(iPSC) succeed in $2006^{1}$, several more CS transition methods have been established both in vivo and in vitro ${ }^{2-4}$, spurring prospective development in biomedical area. However, reprogrammed cells may fail to strictly match in vivo counterparts' identities ${ }^{5}$, and precise reprogramming toward cell subtypes or states remains impossible ${ }^{6}$. As gene expression profiles(GEPs) for each individual cell in query population became accessible with scRNA-seq, substantial progress in accurately defining cell subtypes and states with marker gene expression has been made ${ }^{7,8}$. Multiple computational methods, like CellNet ${ }^{9}$, method of D'Alessio et al ${ }^{10}$, and Mogrify $^{11}$, are available and proved informative of determining key transcription factors(TFs) for CS conversions via carrying out novel human cell conversion experiments. But limited generalized method could achieve similar goal while utilizing scRNA-seq based expression profiles instead of microarray based or Cap analysis gene expression (CAGE) based databases. Indeed, constructing scRNA-seq based gene expression database is possible to expand existing methods' applicability in predicting key TFs for CS conversions and decoding detailed mechanism behind cell reprogramming. Maintaining complicated database covering majority discovered CSs require massive work; hence, developing generalized scRNA-seq based predictive computational method for determining key GFs in CS conversion with existing methods remain challenging. In 2019, SingleCellNet ${ }^{12}$ was published as successor of CellNet in classifying cellular samples with scRNA-seq data but not guiding CS conversion.

Here we present Odysseia, a scRNA-seq based expression profile analysis system utilizing interpretable machine learning classifiers, for guiding CS conversion method design and elucidating mechanisms behind CS conversions. Unlike previously described methods, Odysseia does not require any background expression database but searching for potential key GFs in converting one CS to another with only expression profiles labeled with binary CS categories as input. Genetic regulatory pathways(GRPs) significantly contributing to classifying CS categories will be addressed through assessing and interpreting classification models trained with input data. Then, key genes that likely induce CS conversion will be addressed through analyzing regulons constructed with the important GRPs described above. This design is based on the assumption that the key genes and GRPs may not have converged profiles in either expression or regulatory aspects. Thus, classification model designed to directly find CS deterministic genes or GRPs may fail to converge on a stable solution. One of the intuitive approach to solve the non-convergence issue is transforming previous question into classifying CSs, a converged and labeled profile among input data, with given scGEPs. Then, Odysseia analyzes the well-performing classifiers and find features, equivalent to GRPs, essential to correctly classify scGEPs. 
However, if features associated with similar functions are sufficiently informative, CS classifiers could reach remarkably high accuracy with a small number of heavily-weighted features. As a result, potential key features will presumably be lost even classifiers demonstrated high accuracy over testing data, and output informativity will be impaired.

For example, cell differentiation or CS conversion is accompanied by a series of GEP and epigenetic changes, and these processes usually occur during the cell cycle. ${ }^{13,14}$ As a result, GFs related to cell cycle or proliferation can be used as differential characteristics to classify CSs but are not key regulators of the differentiation process. If only these GFs are found after classifier analysis, system output's capability in guiding CS conversion may not be sufficient. Therefore, a particular classification model designed solving transformed question can still encounter feature lost issue.

In order to overcome feature lost issue, Odysseia applies multiple classification models with distinct architecture settings in each training iteration then selects well-performing classifiers for further assessment and interpretation. Through interpreting top-performing classifiers trained with different batches of input data, Odysseia enhances the feature extraction capability.

Further details and testing results are discussed in the remainder of this paper which is structured as follow:

- The Method section explains the overall system design.

- The Result section shows system performance on three existing datasets with high fidelity.

- At last, further experiments and potential improvements is discussed in the Discussion section.

\section{Method}

Briefly, Odysseia consists of four main steps as shown in Figure 1:

- Step 1: Generate pseudo-celluar gene expression profiles(pseudo-cGEPs) and corresponding pseudo-cellular genetic regulatory network(pseudo-cGRN) with genetic regulatory network(GRN) reconstruction guidance.

- Step 2: Train classification models with GRNs reconstructed with pseudo-cGEPs then select out well-performing models via accuracy evaluation.

- Step 3: Interpret selected classifiers' correct predictions on all generated GRNs.

- Step 4: Perform regulon based analysis on important GRPs found from classifer interpretation in step 3 and extract key genes indicated by the GRPs.

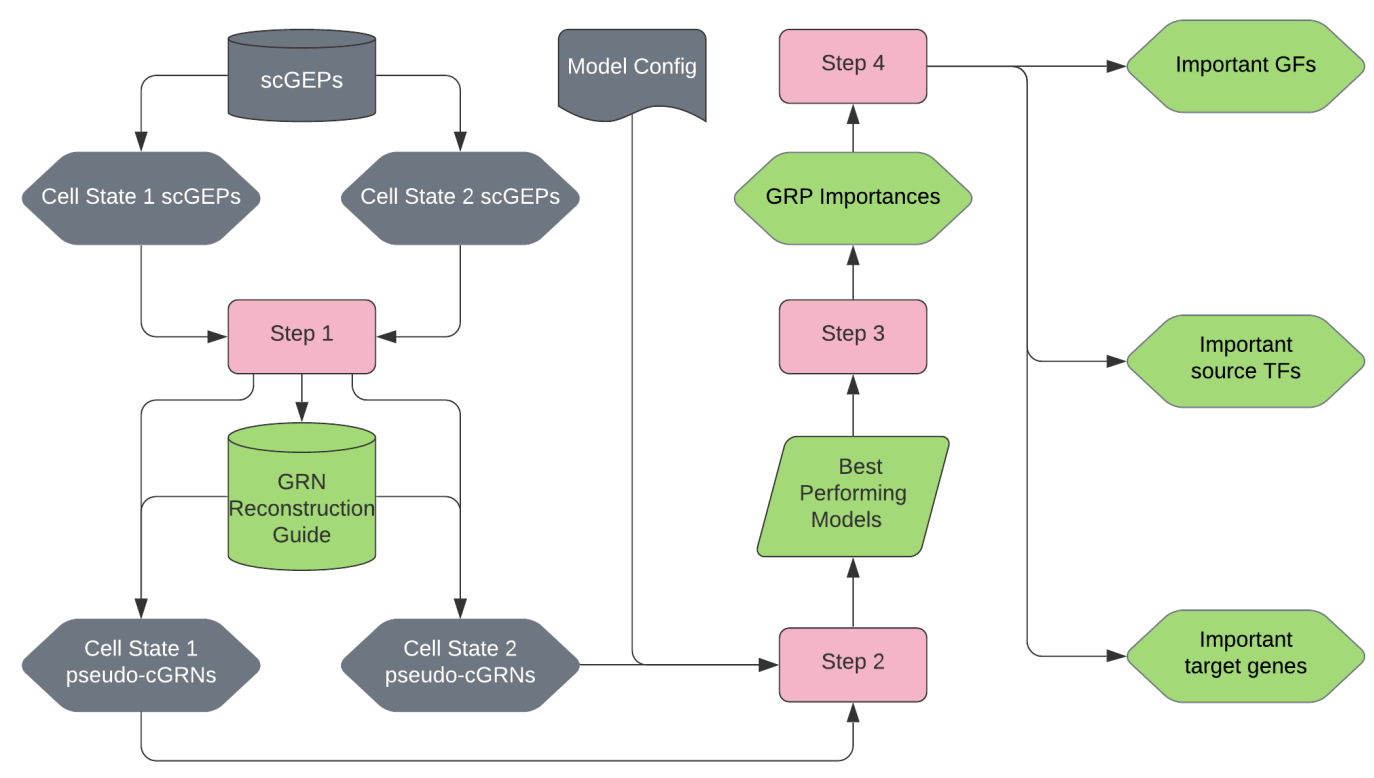

Figure 1. The overall workflow of Odysseia 


\section{Step 1: Data pre-processing}

To reconstruct GRNs based on scGEPs, it is necessary to measure interaction strength among gene pairs. One of the widely adopted methods is Pearson's Correlation coefficient(PCC ${ }^{15,16}$. Nevertheless, calculating PCC requires sequence of expression levels(ELs) for each gene amomg interaction rathar than single EL scRNA-seq can provide for one cell. Hence, in order to reconstruct cell-level GRNs, Odysseia segments scGEPs under same CS category into subsets with constant size to generate pseudo-cGEPs. More specifically, in this step, ELs of one gene from different cells are abstracted as a sequence of temporal changing ELs of corresponding gene in one pseudo cell.

When scGEPs scarece, sliding window algorithm(SWA) with customized window size and padding stride can be applied to ensure sufficient pseudo-cGEP for later classifier training and assessment processes. With SWA, $i$-th pseudo-cGEP can be obtained through:

$$
S W A(i)=\left\{x_{j=i * s}^{j+l}\right\}, j+l<N
$$

Here $N$ denotes total length of scGEPs gained from scRNA-seq, and scRNA-seq can be expressed as $\left\{x_{j}^{N}{ }_{j=0}^{N}\right\} ; l$ denotes window size; $s$ denotes padding stride.

To reduce computational resource requirement, a meta-level process on determining gene pairs might form regulatory relationships in each pseudo-cGRN can dramatically improve efficiency from repeatedly testifying all possible gene pairs in every pseudo-cGEP. Assuming that pseudo-cGEPs have similar expression pattern with comprehensive GEP containing all scGEPs, the GRN reconstruction guidance can be created through analyzing all gene pairs in comprehensive GEP. The overall workflow to create GRN reconsturction guidance is summarized in Figure 2. In total, two sets of filters are used for validating a GRP:

\section{Gene level:}

The filter set below determines binary CS category differentiability of single gene expression pattern.

- SD Filter: The standard deviation(SD) of query gene's ELs must be significant. This filter aims to confirm query gene can both reach relatively high EL and possibly interact with other GFs. SD threshold is set to 1 by default, implying that only genes with expression status changing in different circumstances can be considered. In reality, SD threshold need to be adjusted in response to expression change weight and computational resource capacity.

- MWU Filter: The expression distribution of selected gene across samples labeled with different CSs must be dissimilar. This filter aims to confirm that query gene's GEP is likely distinguishable across sample sets under binary CS categories. The Mann-Whitney U rank test(MWU) implemented by SciPy ${ }^{17}$ will be performed on GEPs of selected gene in binary CS categories. The p-value for rejecting null hypothesis, that expression pattern underlying CS1 is the same as the expression pattern underlying CS2, is set to 0.05 by default.

\section{Pathway level:}

The filter set below determines the biological interpretability and significance of a GRP.

- TF Filter: The source input of a GRP must be a recorded TF. If not further specified, TF list will be retrieved from integrated TRANSFAC ${ }^{18}$ datasets according to the species information in query.

- GRP Filter: Regulatory target must have binding ability with source TF. If not further specified, regulatory gene list of given TF will be retrieved from integrated $G T R D^{19}$ datasets according to the species information in query.

- PCC Filter: Expression correlation between source TF and target gene must be significant. The absolute value threshold of PCC is 0.2 by default, as calculated with $S c i P y^{17}$.

Utilizing GRN reconstruction guidance, pseudo-cGRN will be reconstructed for each pseudo-cGEP. Each gene in the guidance, if also presents in pseudo-cGEP, need to pass the SD filter in pseudo-cGEP circumstance before validating correspond GRPs in the guidance. The pre-validated GRPs that also passes PCC filter will take part in eventual pseudo-cGRN.

\section{Step 2: Classifier selection}

According to given model config file, vanilla models will be initialized with specified model type and architecture parameters. Odysseia will perform accuracy tests systematically aiming to select out potentially well-performing classifiers for further analysis, while iterativly training classifiers with split pseudo-cGRN sets. The overall workflow is summarized in Figure 4. Currently, Odysseia supports three types of classification models:

- SVC: Support Vector Classifer implemented with scikit-learn. ${ }^{20}$ All parameters adjustable with scikit-learn ${ }^{20}$ are supported. 


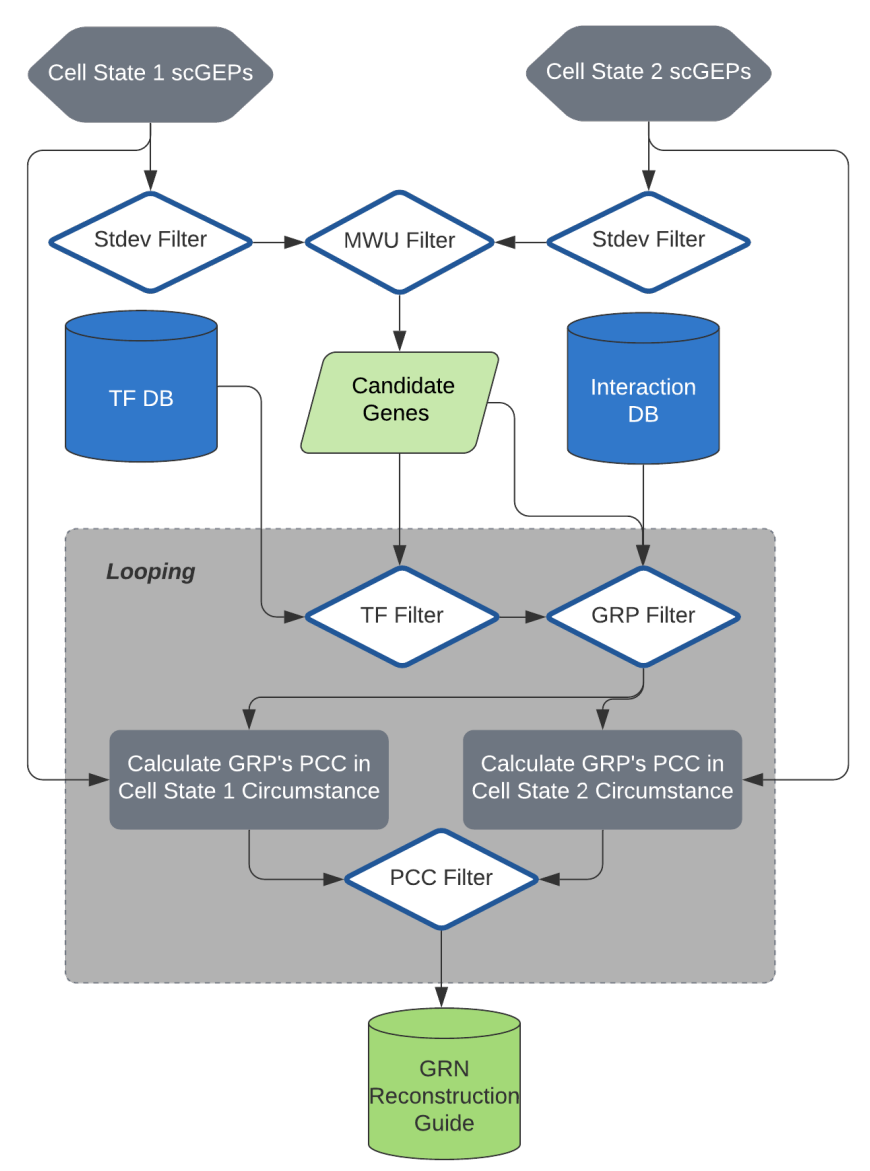

Figure 2. Workflow to create GRN reconstruction guidance from comprehensive GEPs. (1) Each gene in scGEP set either for CS1 or CS2 need to pass SD filter to confirm its expression pattern reacts with inconstant gene expression circumstances. (2) GEPs of genes passed SD filter will be inputted to MWU filter to confirm that its gene expression pattern is differentiable among binary CS categories. (3) All TFs in genes passed MWU filter will be selected out as potential regulatory source TF utilizing given TF database. (4) Using GF or protein-protein interaction databae, all genes passed MWU filter will be testified as target genes with potential TF found at previous step to form potential interacting gene pairs. (5), PCCs of potential interacting gene pairs found previously will be calculated under each CS category. A gene pair must have corresponding PCC under at least one CS category greater than the threshold to be considered as potential GRP.

- GBTree: Gradient Boosting Trees implemented with XGBoost. ${ }^{21}$ All parameters adjustable with XGBoost ${ }^{21}$ are supported.

- CNN: Convolutional Neural Network implemented with Pytorch ${ }^{22}$.

More specifically, the general architecture designs of Odysseia's integrated CNNs are implemented referring to 1D-CNN and 2D-Hybrid-CNN applied in recent cancer type prediction study ${ }^{23}$. Unlike original 1D-CNN and 2D-Hybrid-CNN, we implemented the models with flexibilities on not only kernel related parameters but also others including amount of convolution layer set which consists of a convolution layer and adjacent max-pooling layer. An example of 1D-CNN with 2 convolution layer sets is illustrated in Figure 3.

Other CNNs implemented with Pytorch ${ }^{22}$ can also be analyzed with Odysseia but training protocol need to be clarifed by user in advance.

Eighty one model builds consisting of 1 SVC, 16 GBTrees, and 64 CNNs will be used to initialize vanilla models with default model config file. In each training iteration, each vanilla classifier will be fed with same set of pseudo-cGRNs as training samples and is primarily assessed according to prediction accuracy on untrained pseudo-cGRNs. By default setting, 70\% of pseudo-cGRNs will be split into training set while $30 \%$ will be split into testing set at each iteration. Classifiers capable of passing accuracy threshold will be kept for further comparisons with other classifers trained with different sample sets in later 


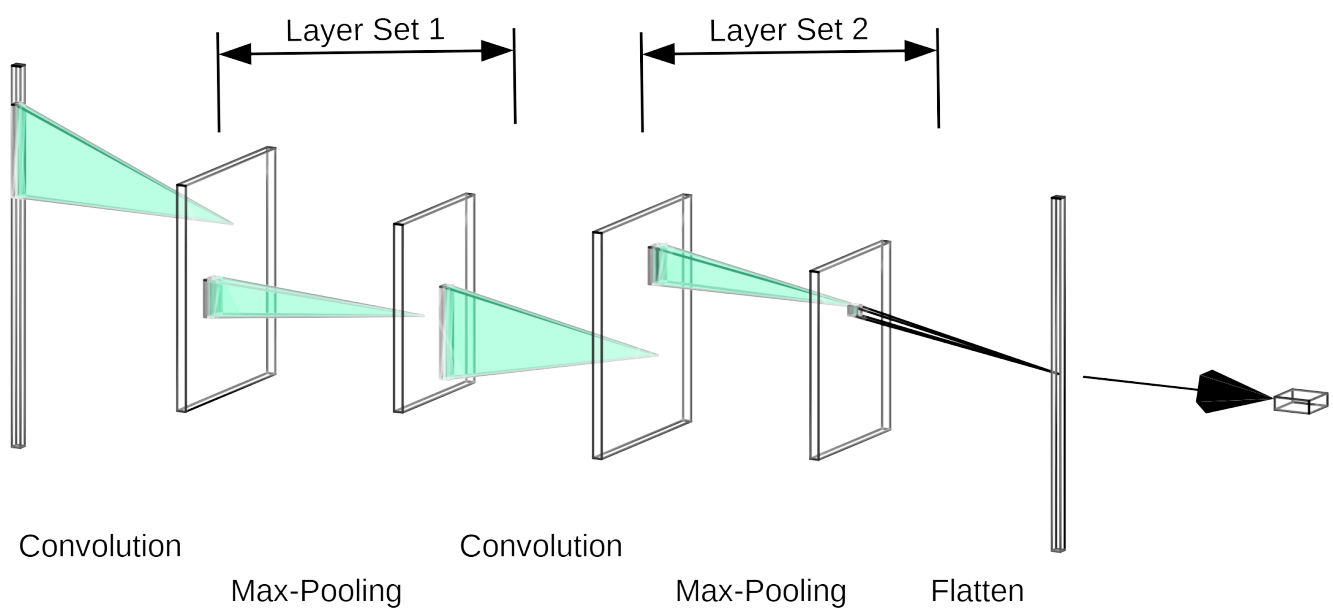

Figure 3. 1D-CNN with 2 convolution layer set.

iterations.

At the end of this step, all available pseudo-cGRNs will be joint to use as testing data, and all candidate classifers will be evaluated on their prediction accuracies of fully joint testing data. Considering that the real random choice can reach an accuracy of 50\% in binary classification question, the accuracy threshold for either local accuracy test or fully joint data test is set to $90 \%$.

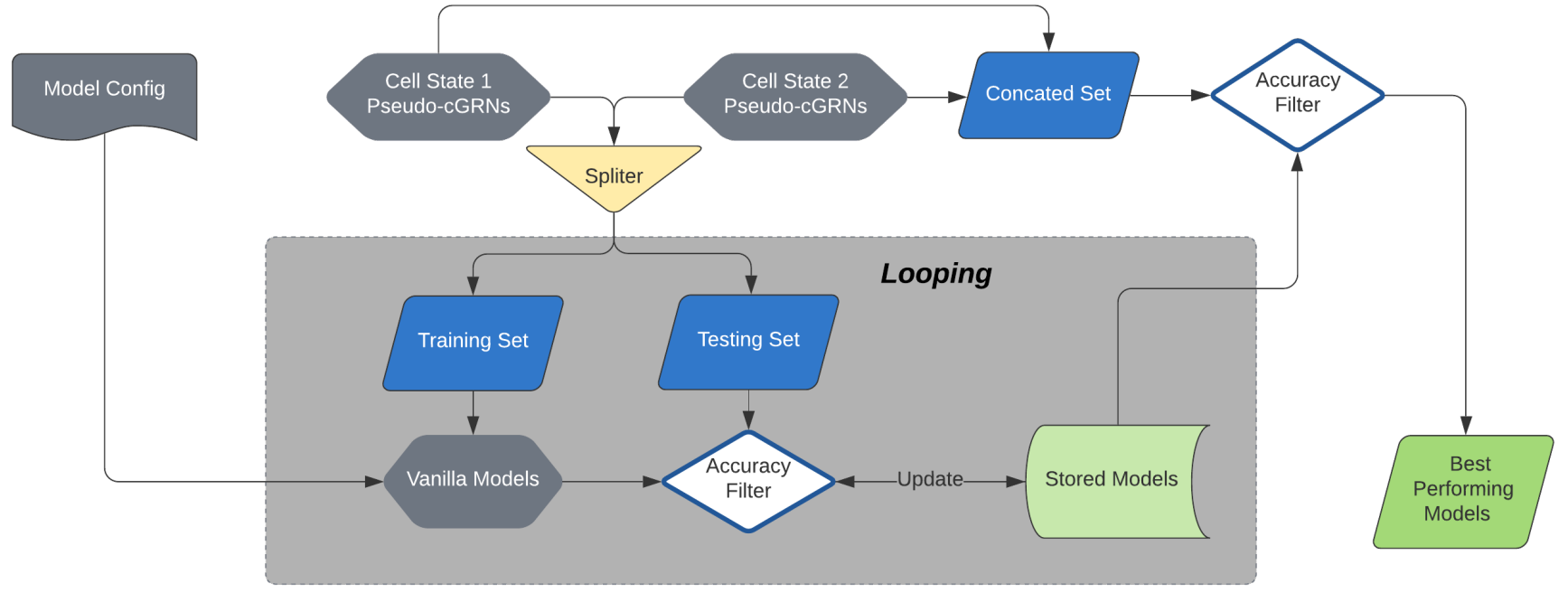

Figure 4. Workflow to generate well-performing classifers. (1) In each training iteration, input pseudo-cGRNs will be split into training set and testing set according to given ratio. By default, the ratio is set to $70 \%$ for training and $30 \%$ for testing. (2) According to given classification model config file, vanilla classifiers will be initialized and trained with training set. The predictive accuracy of classifiers will be pre-assessed with testing data. Classifers reaching accuracy threshold, which is set to 0.9 by default, will be kept for accuracy assessment in further training iterations. (3) After all training iterations, all pseudo-cGRNs will be concatenated as one testing set to assess accuracies of classifers being kept. Classifers reach accuracy threshold, set to 0.9 by default, will be passed to further analysis.

\section{Step 3: Classifier interpretation}

During the last accuracy assessment in Step 2, all classifer's predictions consisting with ground truths will be marked with corresponding input pseudo-cGRNs. Briefly, in this step, Odysseia interprets how well-performing classifiers make correct predictions on CS categories of given pseudo-cGRNs. Integrating interpretations of all well-performing classifers, the 
generalized importance values of features, which are represented by GRPs in Odysseia's scenario, in determining CS can be estimated.

Several packages already have internalized methods implemented for machine learning models to analyze feature importance. For example, GBTree implemented with XGBoost ${ }^{21}$ can have feature importance approximated with average weight gain at each split involving the feature, and linear SVC implemented with scikit-learn ${ }^{20}$ can have the importance estimated with feature coefficient.

Regarding the standard differences between these internalized methods, we utilize softmax function to normalize feature importance and define the normalized importance calculation function as:

$$
T(Z)=\left\{\frac{f\left(Z_{i}\right)}{\sum_{j=1}^{L} f\left(Z_{j}\right)}\right\}, i \in Z
$$

Here $Z$ is feature set; $L$ is total number of features; $f(X)$ is internalized feature importance calculating function of classification model.

However, for numerous machine learning models, there is no internalized feature importance estimating method, for example, NN-based models implemented with Pytorch ${ }^{22}$ or generalized method cannot be appropriately applied with selected models such as Kernelized SVC implement with scikit-learn ${ }^{20}$. Therefore, we apply the concept of The Shapley value ${ }^{24}$ to build an universal approach for determining importance of each feature in any kind of machine learning model. Specific Shapley value calculation or approximating methods are implemented with $S H A P^{25}$ and applied to different types of classifiers as shown in Table1.

\begin{tabular}{|l|l|l|}
\hline Model Genera & Model Example & SHAP $^{25}$ Method \\
\hline Tree-Based & GBTree & Tree Explainer \\
\hline NN-Based without Repetitive Layer Sets & CNN & Deep Explainer \\
\hline NN-Based with Repetitive Layer Sets & Recurrent Neural Network & Gradient Explainer \\
\hline Kernel Machines & SVC & Kernel Explainer \\
\hline Linear Models & Logistic Regression & Linear Explainer \\
\hline
\end{tabular}

Table 1. Machine learning model generas with applicable Shapley value approximating methods

Let $N$ denotes the total number of correct predictions, $\phi_{x, j}^{i}$ and $\phi_{y, j}^{i}$ denotes Shapley value of feature $i$ when predicting sample $j$ as type $x$ or type $y$, the function determining importance values of feature set $z$ can be expressed as:

$$
T(Z)=\operatorname{softmax}\left(\left\{\sum_{j=1}^{N} \frac{\left|\phi_{x, j}^{i}\right|+\left|\phi_{y, j}^{i}\right|}{2}\right\}, i \in Z\right)
$$

Following the analysis of all classifers, each feature's general importance value can be obtained through:

$$
g(i)=\sum_{j=1}^{C} T_{j}(Z)[i]
$$

Here $i$ denotes ith feature or GRP, $C$ denotes the total number of classifers being analyzed, $T_{j}(Z)[i]$ denotes ith feature's importance value calculated through analyzing $\mathrm{j}$ th classifier.

\section{Step 4: Key genes extraction}

With feature list ranked according to importance values, Odysseia reconstructs regulon for top-ranked GRPs and extracts influential genes among the regulon. If analoging regulon with the graph concept in discrete mathematics, one of the most common methods to analyze influence of a vertex, which is equivalent to a gene, is assessing correspond degree of the vertex. Although the regulatory source and target of GRP can be determined by utilizing interaction database, such as GTRD ${ }^{19}$ which shows regulatory relationship of interactors, numerous computational prediction methods like GRNBoost ${ }^{26}$ and comprehensive interaction database, BioGRID ${ }^{27}$ for example, could not provide faithful indication on regulatory directions. Consequently, based on choice of interaction database, the regulon reconstructed with Odysseia cannot be guaranteed to be a directed graph but an undirected graph. Therefore, in this step, odysseia primarily extracts genes with high regulatory degrees in the regulon, regardless of they act as source or target in GRPs. To limit total amount of output key genes, the default setting of Odysseia reconstructs regulon for top 100 GRPs, and genes with regulatory degree higher than two in the regulon will be considered influential. If regulatory directions can be clarifed, the regulatory sources capable of regulating multiple key genes and the regulatory targets influenced by multiple key genes can also be extracted according to GRN reconstruction guidance.

In the circumstance of finding genes potentially inducing CS conversion, referred as Mogrify ${ }^{11}$, both CS deterministic genes and common regulators of these genes shall gain attention. Herein, Odysseia analogizes key genes extracted from top GRPs as CS deterministic genes and TFs capable of regulating more than 2 listed key genes as significant common regulator. 


\section{Results}

In order to evaluate analytic power, we tested Odysseia with three previously published CS conversion related scRNA-seq datasets. Considering the differences in information availability and data size among datasets, each dataset is analyzed with adjusted process.

\section{MEF and ESC}

This dataset was used to study cell fate continuum when reprogramming MEF into iPSC, one of the most well-studied CS conversions. $^{28}$ To simulate the process of finding gene combination for reprogramming somatic cell into pluripotent stem cell, we used scGEPs labeled MEFs and embryonic stem cells(ESCs) as input of Odysseia. Due to the limited amount of scGEPs, SWA with window size set to 10 and padding stride set to 1 was applied to generate 73 ESC pseudo-cGEPs and 65 MEF pseudo-cGEPs. SD threshold is set to 100, which resulted in GRN reconstruct guidance consisting 72440 GRPs, considering the computational load our testing device could accept. While all other parameters remained as default, Odysseia extracted 16 key genes and 9 common regulators. Partial key genes which have already been reported in other studies are listed below, and complete output gene list is included in Supplementary Table 1.

\begin{tabular}{|l|l|l|l|}
\hline Gene & Occurrence & Degree & Previous report \\
\hline Nanog & 16 & 0 & Can reprogram MEF into iPSC with other GFs ${ }^{29-31}$ \\
\hline Esrrb & 10 & 0 & Can reprogram MEF into iPSC with other GFs ${ }^{29,32-34}$ \\
\hline Sall4 & 10 & 0 & Can reprogram MEF into iPSC with other GFs ${ }^{29,34}$ \\
\hline Tfcp211 & 8 & 0 & Key regulatory mediator supporting ESC identity \\
\hline Pou5f1 (Oct4) & 7 & 0 & Can reprogram MEF into iPSC with other GFs \\
\hline Fos & 3 & 2 & Subunit of AP-1 which can prohibit iPSC reprogramming \\
\hline Klf4 & 2 & 0 & Can reprogram MEF into iPSC with other GFs \\
\hline
\end{tabular}

Here, Occurrence refers to occurrences in top ranked GRPs; Degree refers to amount of regulating key genes; Previous report summarizes key findings from previous reports.

\section{MEF and iMPC}

The main purpose of analyzing this dataset is to assess Odysseia's performance while stimulating natural cells with artificially induced cells. This dataset is retrieved from research project studying detailed processes of fibroblast to myocyte and myogenic progenitor cell(MPC) conversion. ${ }^{41}$ For approaching muscle cell reprogramming, the output goal of this analysis is designed to find key genes for converting MEF into myoblast and further derived myotube which can be stimulated with MyoD+ induced MPCs(iMPCs) ${ }^{42}$. Practically, only iMPC scGEPs with EL of Myod1 greater than 1, the lowest positive EL in dataset, were extracted as myoblasts and myotubes co-culture and analyzed with MEF scGEPs. Due to computational limitation on our device, pseudo-cGEPs were generated with every 100 scGEPs. As a result, in total of 99 MyoD+ iMPC and 62 MEFs pseudo-cGEPs were generated. SD threshold was also set to 5 to limit the size of GRN reconstruct guidance to be 3756 GRPs while not automatically passing Myod1. Without any change on other default parameters, Odysseia extracted 6 key genes and 3 common regulators. Partial analysis result is listed below under same format as mentioned in section above, and complete output gene list is included in Supplementary Table 2.

\begin{tabular}{|c|c|c|c|}
\hline Gene & Occurrence & Degree & Previous report \\
\hline Sox4 & 73 & 2 & Myogenesis mediator ${ }^{43,44}$ \\
\hline Mef2c & 23 & 3 & Synergy factor of MyoD in the conversion of MEF into myoblast ${ }^{45,46}$ \\
\hline Myog & 2 & 4 & Marker of differentiating myoblast ${ }^{47,48}$ \\
\hline Tcf4 & 2 & 2 & Marker of MEF in muscle connective tissue; Myogenesis mediator ${ }^{49-51}$ \\
\hline Mef2a & 0 & 3 & Synergy factor of MyoD in the conversion of MEF into myoblast ${ }^{45,46}$ \\
\hline Myod1 & 0 & 3 & Can reprogram MEF into myoblast and myotube solely $y^{41,52,53}$ \\
\hline
\end{tabular}

\section{Radial glia and Neuron}

Furthermore, contemplating feasible difficulty in obtaining purified scRNA-seq dataset of cell subtypes and CSs, we tested Odysseia when analyzing differences between purified and mixed CS data. The input dataset of this analysis consists of scGEPs of purified neurons and iPSC-derived radial glial cells, the progenitor cell of neuron, mixing with neurons. All of the data are retrieved from published dataset used to examine CS conversion from iPSC to neuron. ${ }^{54}$ Similar with MEF and iMPC analysis, pseudo-cGEPs were also generated with every $100 \mathrm{scGEPs}$ which result in a total of 90 neural co-culture and 72 
purified neuron pseudo-cGEPs. Without changes on other default parameters, 40441 GRPs were included in GRN reconstruct guidance, and Odysseia extracted 17 key genes and 19 common regulators. Partial analysis result is listed below under same format as mentioned in section above, and complete output gene list is included in Supplementary Table 3.

\begin{tabular}{|l|l|l|l|}
\hline Gene & Occurrence & Degree & Previous report \\
\hline Ets2 & 48 & 12 & Cell cycle regulator need to be repressed for primary neurogenesis $^{55,56}$ \\
\hline Is11 & 23 & 4 & Key gene for motor neuron development $^{57-59}$ \\
\hline Ascl1 & 16 & 13 & Key neurogenesis regulator $^{54,60}$ Can induce neuronal reprogramming solely $^{61,62}$ \\
\hline Nr3c1 & 0 & 9 & Cell cycle inhibitor during embryonic neurogenesis $^{63}$ \\
\hline Rbpj & 0 & 8 & Key mediator of Notch signaling in neurogenesis $^{64-66}$ \\
\hline Neurod1 & 0 & 7 & Key neurogenesis regulator \\
\hline
\end{tabular}

Even though Odysseia is unable to guarantee a gene combination inducing CS conversion, numerous previously discovered key genes can be successfully extracted, which indicated gene combination known capable to convert CSs. For example, when analyzing MEF with iPSC, the top three genes having most occurrences among important GRPs are determined as key factors to reprogram MEF into iPSC with 7F combination ${ }^{29}$. With Jdp2, which is selected to block AP-1 activity also as Fos, an AP-1 subunit, can indicate, the combination of Jdp2, Nanog, Esrrb, and Sall4 is demonstrated as a minimum 4F functional set to perform cell reprogramming ${ }^{29}$. While Sall4 indicating interaction between Sox 2 and Oct $4^{71}$, main components among Yamanaka factors ${ }^{1,39}$ can also be extracted with Odysseia's analysis result. Between MEF and muscle cell, multiple works demonstrated capability of Myod1, which is successfully extracted by Odysseia, in artificially induce CS conversion. ${ }^{52,53}$ For neurogenesis analysis, Ascl1, included as key factor in several neuronal conversion methods ${ }^{61,62}$, is marked as key gene with the most consequential regulatory influence by Odysseia. It is noteworthy that other factors extracted with Odysseia may also play an important role in CS conversions but have not yet been testified with adequate experiments.

\section{Discussion}

As scRNA-seq technology becomes increasingly accessible and applicable, an efficient and robust analytical system capable of extracting key GFs differentiating CSs will also become highly demanded. In this paper, we showed Odysseia's applicability in analyzing scRNA-seq derived data for further CS-related research. According to test cases so far, key genes significant enough to induce CS conversions appear to have either high occurrence in top ranked GRPs or high regulatory influence on other key genes. Without any previous knowledge on studying CSs, Gene Ontology (GO) enrichment analysis can potentially narrow down key cellular functions changed in different CSs.

However, further tests and optimizations are necessary to confirm Odysseia's applicability on guiding frontier researches. While more resources become available, other classification models and interpretation methods will be added and tested with current version on larger scales. Further optimized model selection process will also be critical to improve system's overall performance.

With result achieved so far, we anticipate that Odysseia will become useful in providing insightful advices on not only learning differences between CSs and cell subtypes, but also finding key GFs to induce CS conversions unachievable so far.

\section{References}

1. Takahashi, K. \& Yamanaka, S. Induction of pluripotent stem cells from mouse embryonic and adult fibroblast cultures by defined factors. Cell 126, 663-676, DOI: 10.1016/j.cell.2006.07.024 (2006).

2. Buganim, Y. et al. Direct reprogramming of fibroblasts into embryonic sertoli-like cells by defined factors. Cell Stem Cell 11, 373-386, DOI: 10.1016/j.stem.2012.07.019 (2012).

3. Qian, L. et al. In vivo reprogramming of murine cardiac fibroblasts into induced cardiomyocytes. Nature 485, 593-598, DOI: 10.1038/nature11044 (2012).

4. Sekiya, S. \& Suzuki, A. Direct conversion of mouse fibroblasts to hepatocyte-like cells by defined factors. Nature DOI: 10.1038/nature10263 (2011).

5. Earley, A. M., Burbulla, L. F., Krainc, D. \& Awatramani, R. Identification of ascl1 as a determinant for human ipsc-derived dopaminergic neurons. Sci. Reports 11, DOI: 10.1038/s41598-021-01366-4 (2021).

6. Wang, H., Yang, Y., Liu, J. \& Qian, L. Direct cell reprogramming: Approaches, mechanisms and progress. Nat. Rev. Mol. Cell Biol. 22, 410-424, DOI: 10.1038/s41580-021-00335-z (2021). 
7. Baron, M. et al. A single-cell transcriptomic map of the human and mouse pancreas reveals inter- and intra-cell population structure. Cell Syst. 3, DOI: 10.1016/j.cels.2016.08.011 (2016).

8. Treutlein, B. et al. Reconstructing lineage hierarchies of the distal lung epithelium using single-cell rna-seq. Nature 509, 371-375, DOI: 10.1038/nature13173 (2014).

9. Cahan, P. et al. Cellnet: Network biology applied to stem cell engineering. Cell 158, 903-915, DOI: 10.1016/j.cell.2014. 07.020 (2014).

10. D'Alessio, A. C. et al. A systematic approach to identify candidate transcription factors that control cell identity. Stem Cell Reports 5, 763-775, DOI: 10.1016/j.stemcr.2015.09.016 (2015).

11. Rackham, O. J. et al. A predictive computational framework for direct reprogramming between human cell types. Nat. Genet. 48, 331-335, DOI: 10.1038/ng.3487 (2016).

12. Tan, Y. \& Cahan, P. Singlecellnet: A computational tool to classify single cell rna-seq data across platforms and across species. Cell Syst. 9, DOI: 10.1016/j.cels.2019.06.004 (2019).

13. Dalton, S. Linking the cell cycle to cell fate decisions. Trends Cell Biol. 25, 592-600, DOI: 10.1016/j.tcb.2015.07.007 (2015).

14. Engström, Y. Cell cycle regulators control stemness and differentiation. BioEssays 43, 2100123, DOI: 10.1002/bies. 202100123 (2021).

15. Liu, L.-y. D., Hsiao, Y.-C., Chen, H.-C., Yang, Y.-W. \& Chang, M.-C. Construction of gene causal regulatory networks using microarray data with the coefficient of intrinsic dependence. Bot. Stud. 60, DOI: 10.1186/s40529-019-0268-8 (2019).

16. Song, L., Langfelder, P. \& Horvath, S. Comparison of co-expression measures: Mutual information, correlation, and model based indices. BMC Bioinforma. 13, DOI: 10.1186/1471-2105-13-328 (2012).

17. Virtanen, P. et al. SciPy 1.0: Fundamental Algorithms for Scientific Computing in Python. Nat. Methods 17, 261-272, DOI: 10.1038/s41592-019-0686-2 (2020).

18. Matys, V. Transfac(r) and its module transcompel(r): Transcriptional gene regulation in eukaryotes. Nucleic Acids Res. 34, DOI: $10.1093 / \mathrm{nar} / \mathrm{gkj} 143$ (2006).

19. Kolmykov, S. et al. GTRD: an integrated view of transcription regulation. Nucleic Acids Res. 49, D104-D111, DOI: 10.1093/nar/gkaa1057 (2020). https://academic.oup.com/nar/article-pdf/49/D1/D104/35364856/gkaa1057.pdf.

20. Pedregosa, F. et al. Scikit-learn: Machine learning in Python. J. Mach. Learn. Res. 12, 2825-2830 (2011).

21. Chen, T. \& Guestrin, C. Xgboost: A scalable tree boosting system. In Proceedings of the 22nd acm sigkdd international conference on knowledge discovery and data mining, 785-794 (2016).

22. Paszke, A. et al. Pytorch: An imperative style, high-performance deep learning library. In Wallach, H. et al. (eds.) Advances in Neural Information Processing Systems 32, 8024-8035 (Curran Associates, Inc., 2019).

23. Mostavi, M., Chiu, Y.-C., Huang, Y. \& Chen, Y. Convolutional neural network models for cancer type prediction based on gene expression - bmc medical genomics (2020).

24. Roth, A. E. \& Shapley, L. S. The shapley value : essays in honor of lloyd s. shapley. Economica 101, 123 (1991).

25. Lundberg, S. M. \& Lee, S.-I. A unified approach to interpreting model predictions. In Proceedings of the 31st international conference on neural information processing systems, 4768-4777 (2017).

26. Moerman, T. et al. Grnboost 2 and arboreto: Efficient and scalable inference of gene regulatory networks. Bioinforma. (Oxford, England) 35, DOI: 10.1093/bioinformatics/bty916 (2018).

27. Stark, C. Biogrid: A general repository for interaction datasets. Nucleic Acids Res. 34, DOI: 10.1093/nar/gkj109 (2006).

28. Guo, L. et al. Resolving cell fate decisions during somatic cell reprogramming by single-cell rna-seq. Mol. Cell 73, DOI: 10.1016/j.molcel.2019.01.042 (2019).

29. Wang, B. et al. Induction of pluripotent stem cells from mouse embryonic fibroblasts by jdp2-jhdm1b-mkk6-glis1-nanogessrb-sall4. Cell Reports 27, DOI: 10.1016/j.celrep.2019.05.068 (2019).

30. Yu, J. et al. Induced pluripotent stem cell lines derived from human somatic cells. Science 318, 1917-1920, DOI: 10.1126/science.1151526 (2007).

31. Wang, Y. et al. Reprogramming of mouse and human somatic cells by high-performance engineered factors. $E M B O$ reports 12, 373-378, DOI: 10.1038/embor.2011.11 (2011). 
32. Huang, D. et al. Lif activated jak signaling determines esrrb expression during late-stage reprogramming. Biol. Open DOI: 10.1242/bio.029264 (2017).

33. Benchetrit, H. et al. Direct induction of the three pre-implantation blastocyst cell types from fibroblasts. Cell Stem Cell 24, DOI: 10.1016/j.stem.2019.03.018 (2019).

34. Iseki, H. et al. Combined overexpression of jarid2, prdm14, esrrb, and sall4a dramatically improves efficiency and kinetics of reprogramming to induced pluripotent stem cells. Stem Cells 34, 322-333, DOI: 10.1002/stem.2243 (2015).

35. Wang, X. et al. The transcription factor tfcp 211 induces expression of distinct target genes and promotes self-renewal of mouse and human embryonic stem cells. J. Biol. Chem. 294, 6007-6016, DOI: 10.1074/jbc.ra118.006341 (2019).

36. Ye, S., Li, P., Tong, C. \& Ying, Q.-L. Embryonic stem cell self-renewal pathways converge on the transcription factor tfcp211. The EMBO J. 32, 2548-2560, DOI: 10.1038/emboj.2013.175 (2013).

37. Nemajerova, A., Kim, S. Y., Petrenko, O. \& Moll, U. M. Two-factor reprogramming of somatic cells to pluripotent stem cells reveals partial functional redundancy of sox2 and klf4. Cell Death Differ. 19, 1268-1276, DOI: 10.1038/cdd.2012.45 (2012).

38. Shi, Y. et al. Induction of pluripotent stem cells from mouse embryonic fibroblasts by oct 4 and klf4 with small-molecule compounds. Cell Stem Cell 3, 568-574, DOI: 10.1016/j.stem.2008.10.004 (2008).

39. Maekawa, M. et al. Direct reprogramming of somatic cells is promoted by maternal transcription factor glis1. Nature 474, 225-229, DOI: 10.1038/nature10106 (2011).

40. Li, D. et al. Chromatin accessibility dynamics during ipsc reprogramming. Cell Stem Cell 21, 819-833.e6, DOI: https://doi.org/10.1016/j.stem.2017.10.012 (2017).

41. Kim, I. et al. Integrative molecular roadmap for direct conversion of fibroblasts into myocytes and myogenic progenitor cells. bioRxiv DOI: 10.1101/2021.08.20.457151 (2021).

42. Bar-Nur, O. et al. Direct reprogramming of mouse fibroblasts into functional skeletal muscle progenitors. Stem Cell Reports 10, 1505-1521, DOI: https://doi.org/10.1016/j.stemcr.2018.04.009 (2018).

43. Jang, S.-M. et al. Sox4-mediated caldesmon expression facilitates skeletal myoblast differentiation. J. cell science 126, DOI: $10.1242 / j$ cs.131581 (2013).

44. Liu, S. et al. Genome architecture mediates transcriptional control of human myogenic reprogramming. iScience 6, 232-246, DOI: 10.1016/j.isci.2018.08.002 (2018).

45. Taylor, M. V. \& Hughes, S. M. Mef2 and the skeletal muscle differentiation program. Semin. Cell Dev. Biol. 72, 33-44, DOI: $10.1016 /$ j.semcdb.2017.11.020 (2017).

46. Liu, N. et al. Requirement of mef2a, c, and d for skeletal muscle regeneration. Proc. Natl. Acad. Sci. 111, 4109-4114, DOI: 10.1073/pnas.1401732111 (2014). https://www.pnas.org/content/111/11/4109.full.pdf.

47. Andrés, V. \& Walsh, K. Myogenin expression, cell cycle withdrawal, and phenotypic differentiation are temporally separable events that precede cell fusion upon myogenesis. J. Cell Biol. 132, 657-666, DOI: 10.1083/jcb.132.4.657 (1996).

48. Dumont, N. A. \& Rudnicki, M. A. Characterizing satellite cells and myogenic progenitors during skeletal muscle regeneration. Methods Mol. Biol. 179-188, DOI: 10.1007/978-1-4939-6788-9_12 (2017).

49. Mathew, S. J. et al. Connective tissue fibroblasts and tcf4 regulate myogenesis. Development 138, 371-384, DOI: 10.1242/dev.057463 (2011).

50. Contreras, O., Rebolledo, D. L., Oyarzún, J. E., Olguín, H. C. \& Brandan, E. Connective tissue cells expressing fibro/adipogenic progenitor markers increase under chronic damage: Relevance in fibroblast-myofibroblast differentiation and skeletal muscle fibrosis. Cell Tissue Res. 364, 647-660, DOI: 10.1007/s00441-015-2343-0 (2016).

51. Fry, C. S., Kirby, T. J., Kosmac, K., McCarthy, J. J. \& Peterson, C. A. Myogenic progenitor cells control extracellular matrix production by fibroblasts during skeletal muscle hypertrophy. Cell Stem Cell 20, 56-69, DOI: 10.1016/j.stem.2016.09.010 (2017).

52. Choi, J. et al. Myod converts primary dermal fibroblasts, chondroblasts, smooth muscle, and retinal pigmented epithelial cells into striated mononucleated myoblasts and multinucleated myotubes. Proc. Natl. Acad. Sci. 87, 7988-7992, DOI: 10.1073/pnas.87.20.7988 (1990).

53. Chakraborty, S. et al. A crispr/cas9-based system for reprogramming cell lineage specification. Stem cell reports $\mathbf{3}$, 940-947 (2014). 
54. Earley, A. M., Burbulla, L. F., Krainc, D. \& Awatramani, R. Identification of ascl1 as a determinant for human ipsc-derived dopaminergic neurons. Sci. Reports 11, DOI: 10.1038/s41598-021-01366-4 (2021).

55. Janesick, A., Wu, S. C. \& Blumberg, B. Retinoic acid signaling and neuronal differentiation. Cell. Mol. Life Sci. 72, 1559-1576, DOI: 10.1007/s00018-014-1815-9 (2015).

56. Janesick, A. et al. Erf and etv 31 are retinoic acid-inducible repressors required for primary neurogenesis. Development 140, 3095-3106, DOI: 10.1242/dev.093716 (2013).

57. Pfaff, S. L., Mendelsohn, M., Stewart, C. L., Edlund, T. \& Jessell, T. M. Requirement for lim homeobox gene isl1 in motor neuron generation reveals a motor neuron-dependent step in interneuron differentiation. Cell 84, 309-320 (1996).

58. Liang, X. et al. Isl1 is required for multiple aspects of motor neuron development. Mol. Cell. Neurosci. 47, 215-222 (2011).

59. Zhou, M. et al. Reprogramming astrocytes to motor neurons by activation of endogenous ngn2 and isl1. Stem Cell Reports 16, 1777-1791 (2021).

60. Raposo, A. A. et al. Ascl1 coordinately regulates gene expression and the chromatin landscape during neurogenesis. Cell reports 10, 1544-1556 (2015).

61. Chanda, S. et al. Generation of induced neuronal cells by the single reprogramming factor ascl1. Stem cell reports 3 , 282-296 (2014).

62. Robinson, M. et al. Transdifferentiating astrocytes into neurons using ascl1 functionalized with a novel intracellular protein delivery technology. Front. Bioeng. Biotechnol. 173 (2018).

63. Shin, J. et al. Single-cell rna-seq with waterfall reveals molecular cascades underlying adult neurogenesis. Cell stem cell 17, 360-372 (2015).

64. Imayoshi, I. \& Kageyama, R. The role of notch signaling in adult neurogenesis. Mol. neurobiology 44, 7-12 (2011).

65. Fujimoto, M. et al. Rbp-j promotes neuronal differentiation and inhibits oligodendroglial development in adult neurogenesis. Dev. biology 332, 339-350 (2009).

66. Tanigaki, K. \& Honjo, T. Two opposing roles of rbp-j in notch signaling. Curr. topics developmental biology 92, 231-252 (2010).

67. Pataskar, A. et al. Neurod1 reprograms chromatin and transcription factor landscapes to induce the neuronal program. The EMBO journal 35, 24-45 (2016).

68. Gao, Z. et al. Neurod1 is essential for the survival and maturation of adult-born neurons. Nat. neuroscience 12, 1090-1092 (2009).

69. Boutin, C. et al. Neurod1 induces terminal neuronal differentiation in olfactory neurogenesis. Proc. Natl. Acad. Sci. 107, 1201-1206 (2010).

70. Roybon, L. et al. Neurogenin 2 directs granule neuroblast production and amplification while neurod 1 specifies neuronal fate during hippocampal neurogenesis. PloS one 4, e4779 (2009).

71. Tanimura, N., Saito, M., Ebisuya, M., Nishida, E. \& Ishikawa, F. Stemness-related factor sall4 interacts with transcription factors oct-3/4 and sox 2 and occupies oct-sox elements in mouse embryonic stem cells. J. Biol. Chem. 288, 5027-5038, DOI: 10.1074/jbc.m112.411173 (2013).

\section{Author contributions statement}

J.Y.: Methodology, Software, Writing- Original draft preparation J.T.: Writing - Review \& Editing J.W.: Project administration

\section{Additional information}

All scRNA-seq datasets are retrieved from Gene Expression Omnibus(GEO) as described in Key Resource Table below. 
bioRxiv preprint doi: https://doi.org/10.1101/2022 $02.17 .480852 \cdot$ this version posted February 25,2022 . The copyright holder for this preprint (which was not certified by peer review) is the author/funder, who has granted bioRxiv a license to display the preprint in perpetuity. It is made available under aCC-BY-NC-ND 4.0 International license.

\section{Key Resource Table}

\begin{tabular}{|l|l|l|}
\hline Resource & Source & Usage \\
\hline MEF and ESC scRNA-seq data & GEO Accession: GSE103221 & $\begin{array}{l}\text { Samples with label started with 'mef' as MEF } \\
\text { Samples with label started with 'esc' as ESC }\end{array}$ \\
\hline MEF and iMPC scRNA-seq data & GEO Accession: GSE169054 & $\begin{array}{l}\text { GSM5175907 as iMPC } \\
\text { GSM5643793 as MEF }\end{array}$ \\
\hline Radial glia and Neuron scRNA-seq data & GEO Accession: GSE185275 & $\begin{array}{l}\text { GSM5609927 as neural co-culture } \\
\text { GSM5609930 as purified neurons }\end{array}$ \\
\hline
\end{tabular}

